\title{
Development of a Hard X-ray Nanoprobe Beamline at the Advanced Photon Source
}

\author{
Jörg Maser, *** Brian Stephenson, **** Robert Winarski, ${ }^{*}$ Christa Benson, ** Deming Shu, ** Barry \\ Lai, ** Stefan Vogt, ** Martin Holt *** \\ * Center for Nanoscale Materials, Argonne National Laboratory \\ ** Advanced Photon Source, Argonne National Laboratory \\ *** Materials Sciences Division, Argonne National Laboratory
}

The Center for Nanoscale Materials, under construction at Argonne National Laboratory, is one of five Nanoscale Science Research Centers sponsored by the U.S. Department of Energy. Its mission is the creation, characterization, and understanding of novel functional materials and devices. The Center for Nanoscale Materials will house a variety of fabrication, processing, and characterization facilities, including low- and high-voltage electron beam lithography systems, a focused ion beam system, nanoimprint tools, and confocal and scanning probe microscopes. As a major characterization facility, we are constructing a hard x-ray nanoprobe beamline at the Advanced Photon Source [1]. We will report on the design of the beamline, and the design and capabilities of the hard x-ray nanoprobe instrument.

The hard x-ray nanoprobe is designed to characterize nanoscale systems and devices at a spatial resolution of $30 \mathrm{~nm}$ and below. X-ray techniques employed for characterization are X-ray fluorescence and spectroscopy, x-ray diffraction and scattering, and transmission imaging. X-ray fluorescence will provide element-specific imaging with sensitivity to individual nanoparticles embedded in thick specimens. X-ray diffraction and scattering will probe strain state and ordering of nanoscale systems and their environment. Transmission imaging will allow visualization of thick specimens and devices in 3D. To allow x-ray fluorescence spectroscopy of most elements in the periodic system, the beamline will deliver x-rays with photon energies between $3 \mathrm{keV}$ and $30 \mathrm{keV}$. Two collinear insertion devices with a period of $3.3 \mathrm{~cm}$ and a combined length of $4.8 \mathrm{~m}$ are used as source of x-rays. This maximizes the coherent x-ray flux available in the nanoprobe instrument. The beamline optics are designed to allow two modes of operation: a scanning probe mode, where the spatially coherent fraction of the undulator beam is focused by a high-resolution x-ray optic on a small specimen area, and a full-field transmission mode, where the full, partially coherent undulator beam is used to allow transmission imaging at high resolution. Switching between the two modes is provided by changing the size of an aperture in the beamline and by reconfiguring the x-ray optics in the nanoprobe instrument.

The nanoprobe instrument is based on use of Fresnel zone plates as high-resolution x-ray optics. Zone plates will be used as focusing optics in scanning probe mode and as imaging optics in full-field transmission mode. In scanning probe mode, the zone plate is placed upstream of the specimen, and focuses the incident coherent wavefront into a diffraction limited spot on the specimen. Secondary signals excited in the specimen, namely x-ray fluorescence and x-ray diffraction and scattering, are used to characterize the specimen properties. In full-field transmission mode, a condensor system is used to illuminate the specimen with a partially coherent x-ray beam, and the high-resolution zone plate is placed downstream of the specimen, creating a magnified image of the specimen on an area detector. A tomographic image of the specimen is acquired by recording images at different specimen angles. The combination of both operation modes provides both high-resolution imaging of complex devices, and analytic capabilities to study small areas in the same devices. 
The nanoprobe instrument is currently under design. To provide initial x-ray microscopy capabilities before availability of the nanoprobe instrument, an early version of the instrument has been developed [2]. This system is based on a prototype high-precision scanning stage and specimen stage. It is designed to provide a mechanical repeatability of $+/-2 \mathrm{~nm}$ over a travel range of $12 \mathrm{~mm}$ for lateral zone plate and specimen position, and to maintain the lateral position to better than $15 \mathrm{~nm}$ during the focusing motion of the zone plate. First experiments using this instrument will be presented.

\section{References}

[1] J. Maser, G.B. Stephenson, D. Shu, B. Lai, S. Vogt, A. Khounsary, Y. Li, C. Benson, G. Schneider, AIP Conf. Proc. 705, AIP (2004) $470-473$.

[2] D. Shu, J. Maser, B. Lai, and S. Vogt, AIP Conf. Proc. 705, 1287-1290 (2004).

[3] This work was supported by the U.S. Department of Energy, Office of Basic Energy Sciences, under Contract No. W-31-109-ENG-38. We wish to thank G. Schneider for his contributions to the beamline design.

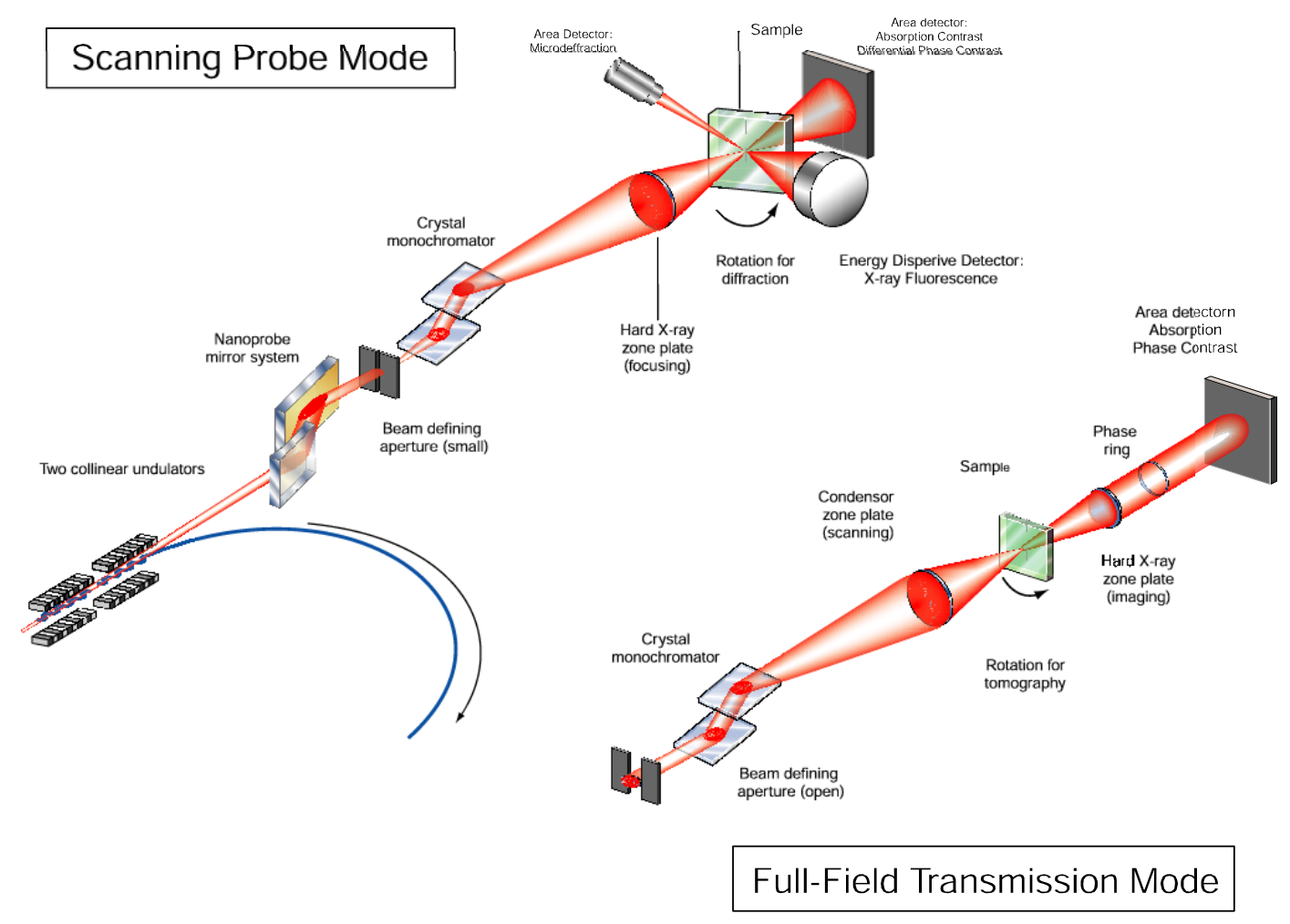

FIG. 1 Scheme of the hard x-ray nanoprobe beamline in scanning probe mode and in full-field transmission mode. In scanning probe mode, the spatially coherent fraction of the undulator beam is focused into a diffraction-limited spot on the specimen. This allows use of secondary signals such as $\mathrm{x}$-ray fluorescence photons and scattered radiation. In full-field transmission mode, a magnified image of the whole specimen field is formed using absorption or phase contrast. Fresnel zone plates are used as high-resolution optics both for focusing and imaging. 